\title{
Four-dimensional symplectic cobordisms containing three-handles
}

\author{
DAVID T GAY
}

\begin{abstract}
We construct four-dimensional symplectic cobordisms between contact three-manifolds generalizing an example of Eliashberg. One key feature is that any handlebody decomposition of one of these cobordisms must involve three-handles. The other key feature is that these cobordisms contain chains of symplectically embedded twospheres of square zero. This, together with standard gauge theory, is used to show that any contact three-manifold of non-zero torsion (in the sense of Giroux) cannot be strongly symplectically fillable. John Etnyre pointed out to the author that the same argument together with compactness results for pseudo-holomorphic curves implies that any contact three-manifold of non-zero torsion satisfies the Weinstein conjecture. We also get examples of weakly symplectically fillable contact three-manifolds which are (strongly) symplectically cobordant to overtwisted contact three-manifolds, shedding new light on the structure of the set of contact three-manifolds equipped with the strong symplectic cobordism partial order.
\end{abstract}

57R17, 53D35; 57M50, 53D20

\section{Main Theorem}

Eliashberg [8] showed that certain contact structures $\xi_{n}$ on $T^{3}$, although weakly symplectically fillable, are not strongly symplectically fillable (for $n>1$ ), by constructing a symplectic cobordism from $\left(T^{3}, \xi_{n}\right)$ to the disjoint union of $n$ copies of the standard contact $S^{3}$. An interesting feature, then, is that this is a cobordism from a connected contact 3-manifold to a disconnected contact 3-manifold and thus necessarily contains a 3-handle. By contrast, most other constructions of symplectic cobordisms from contact 3-manifolds to contact 3-manifolds (as in Etnyre and Honda [11]) are built out of elementary 0-, 1- and 2-handle cobordisms as in Eliashberg [7] or Weinstein [25]. To this author's knowledge there is no model for an elementary contact-to-contact 3-handle symplectic cobordism. Thus it would be interesting to isolate the 3-handle from Eliashberg's $T^{3}$-to- $S^{3}$ cobordisms and then generalize Eliashberg's nonfillability results. We have not succeeded in usefully isolating the 3-handle but we have localized the construction to a certain extent, with new and interesting consequences for 
nonfillability and for the cobordism relation in general. (For background on varieties of fillability and known results, see Etnyre and Honda $[10 ; 11]$.)

Given any real numbers $a>b$, let $M_{a, b}=[a, b] \times S^{1} \times S^{1}$, with coordinates $(t, x, y)$, and consider the contact structure $\xi_{a, b}=\operatorname{ker}(\cos (\pi t) d y+\sin (\pi t) d x)$. Given any $c>0$ let $\left(M_{c}, \xi_{c}\right)=\left(M_{0, c}, \xi_{0, c}\right)$. Also, using "toric coordinates" $(p, q)$ on $\mathbb{R}^{2}$ with $p=r^{2} / 2$ and $q=\theta$, and given any $a>0$, let $T_{a}$ be the solid torus $S^{1} \times\{p \leq a\}$ (with $\alpha$ the $S^{1}$-coordinate), and let $\eta_{a}=\operatorname{ker}(\cos (\pi p) d \alpha+\sin (\pi p) d q$ ).

Our main result is:

Theorem 1 For any $k \in \mathbb{N}$, with $k>1$, there exists a smooth cobordism-withsides $X_{k}$ from $M_{k+1 / 2}$ to $T_{1 / 2} \amalg T_{1 / 2}$ containing a chain of embedded 2-spheres $S_{1}, \ldots, S_{2 k-2}$, with a symplectic form $\omega_{k}$, satisfying the following properties:

- Each $S_{i}$ is symplectic and all intersections between $S_{i}$ 's are positive.

- $S_{i} \cdot S_{j}=0$ unless $j-i= \pm 1$, in which case $S_{i} \cdot S_{i+1}=+1$.

- There is a nowhere-zero Liouville vector field $V$ defined in a neighborhood of the bottom and sides, pointing in along the bottom and parallel to the sides, inducing the contact structure $\xi_{k+1 / 2}$ on the bottom $M_{k+1 / 2}$ (i.e. $\xi_{k+1 / 2}=$ $\left.\operatorname{ker}\left(\left.l_{V} \omega\right|_{M_{k+1 / 2}}\right)\right)$.

- Along the top, the contact structure $\xi_{1 / 2}$ on each copy of $T_{1 / 2}$ is dominated by $\omega_{k}$ (i.e. $\left.\left.\omega_{k}\right|_{\xi_{1 / 2}}>0\right)$, and agrees with $\operatorname{ker}\left(\left.l_{V} \omega\right|_{T_{1 / 2}}\right.$ ) on a neighborhood of $\partial T_{1 / 2}$.

A nearly identical theorem could be stated producing a chain of (fewer) spheres of self-intersection +1 . The version above is slightly more convenient to state and prove because a square (the moment map image of $S^{2} \times S^{2}$ ) is slightly easier to work with in cartesian coordinates than a triangle (the moment map image of $\mathbb{C} P^{2}$ ). We have not stated which curves on $\partial M_{k+1 / 2}$ correspond to meridians in $T_{1 / 2} \amalg T_{1 / 2}$ as we do not need this information for our applications; if needed, this information is extractable from the proof. Also, for the applications in this paper we only need the case $k=2$; presenting only this case would shorten the proof, but we believe that the general result is interesting in its own right so we present it in full.

\section{Corollaries}

Before the proof, we discuss some corollaries. As a prerequisite we have: 
Definition 2 (Giroux $[15 ; 16 ; 17]$ ) Given an isotopy class $C$ of tori in a contact 3 -manifold $(M, \xi)$, the torsion of $C$ is the largest integer $n$ such that $\left(M_{2 n}, \xi_{2 n}\right)$ can be contactomorphically embedded in $(M, \xi)$ as a neighborhood of a torus in $C$.

We will be a bit sloppy, and speak of the torsion of a torus, meaning the torsion of its isotopy class.

Corollary 3 If a contact 3-manifold $(M, \xi)$ contains a torus of torsion greater than or equal to 1 then $(M, \xi)$ is not symplectically fillable.

This was apparently a conjecture of Eliashberg's. It should be noted that Ding and Geiges [4] generalized Eliashberg's result on nonfillability of contact structures on $T^{3}$ to contact structures on general $T^{2}$ bundles over $S^{1}$, and thus proved this corollary in certain very special cases. Ghiggini [14] then proved this for certain Seifert fibred 3-manifolds, and most recently Lisca and Stipsicz [19] proved it for a larger class of 3-manifolds, characterized in terms of their Ozsvath-Szabo invariants.

Proof Note that a neighborhood of $\left(M_{k}, \xi_{k}\right)$ always contains $\left(M_{k+\epsilon}, \xi_{k+\epsilon}\right)$ for small $\epsilon>0$, and thus that a neighborhood of $\left(M_{2}, \xi_{2}\right)$ contains a copy of $\left(M_{2+1 / q}, \xi_{2+1 / q}\right)$ for large enough integer $q$. However, for any $q \in \mathbb{Z},\left(M_{2+1 / q}, \xi_{2+1 / q}\right)$ is contactomorphic to $\left(M_{5 / 2}, \xi_{5 / 2}\right)$ via a contactomorphism of the form $(t,(x, y)) \mapsto(f(t), L(x, y))$ where $f$ is smooth and increasing and $L$ is linear. Thus we now assume that $(M, \xi)$ contains $\left(M_{5 / 2}, \xi_{5 / 2}\right)$.

Suppose $(M, \xi)$ is the strongly convex boundary of a compact symplectic 4-manifold $(X, \omega)$. Then we can attach the cobordism $\left(X_{2}, \omega_{2}\right)$ along $\left(M_{5 / 2}, \xi_{5 / 2}\right)$, attaching a trivial cobordism made from the symplectization of $\xi$ along the rest of $M$, to produce a symplectic 4-manifold $\left(X^{\prime}, \omega^{\prime}\right)$ with (weakly) convex boundary containing a symplectic "hyperbolic pair" $\left(S_{1}, S_{2}\right)$, with $S_{1} \cdot S_{2}=+1=\left|S_{1} \cap S_{2}\right|$ and $S_{1} \cdot S_{1}=$ $S_{2} \cdot S_{2}=0$.

Now cap off $\left(X^{\prime}, \omega^{\prime}\right)$ with a concave filling (see Etnyre [10] or Eliashberg [9]) to get a closed symplectic 4 -manifold. Such a concave filling can always be constructed so that $b_{2}^{+}$of the filling is positive. This is because, if we use the construction in [10], the first step is to construct a cobordism up to a homology 3-sphere (Lemma 3.1 of [10]), at which point the weakly convex boundary can be made into a strongly convex boundary (Ohta and Ono [21]), which can then be capped off with a concave filling as in Gay [12], in which one can explicitly see a surface of positive self-intersection. Thus we have a closed symplectic 4 -manifold with $b_{2}^{+}>1$, containing a hyperbolic pair of spheres, which is well-known to be impossible: Since the boundary of a neighborhood of a 
hyperbolic pair (with geometric intersection equal to algebraic intersection) is $S^{3}$, this means that the 4-manifold splits as a connected sum of two 4-manifolds each with $b_{2}^{+} \geq 1$, which cannot happen because symplectic 4 -manifolds have nontrival Seiberg-Witten invariants (Tuabes [24]) while connected sums of 4-manifolds with $b_{2}^{+} \geq 1$ have trivial Seiberg-Witten invariants (see [21], for example).

The next corollary and the proof presented here were explained to the author by John Etnyre. Recall that the generalized Weinstein conjecture for a contact 3-manifold $(M, \xi)$ states that any Reeb vector field for $\xi$ has a closed orbit.

Corollary 4 (Etnyre) Any contact 3-manifold $(M, \xi)$ containing a torus of non-zero torsion satisfies the Weinstein conjecture.

Note that this is already known to be true on a large class of non-zero torsion contact 3 -manifolds as a result of the computations in Bourgeois and Colin [1].

Sketch of proof As in the preceding proof, we can construct a concave cap for $(M, \xi)$, namely a symplectic 4 -manifold $(X, \omega)$ with strongly concave boundary $(M, \xi)$, containing a symplectic sphere $S$ of square zero. Attaching the negative symplectization of a particular contact form $\alpha$ for $\xi$ gives a noncompact manifold with negative cylindrical end as in Bourgeois, Eliashberg, Hofer, Wysocki and Zehnder [2]. The compactness results in [2] can be used to extend to this setting the techniques which McDuff [20] used to understand closed symplectic 4-manifolds containing square zero symplectic $2-$ spheres. This shows that, if $R_{\alpha}$ has no closed orbits, then the moduli space of $J$-holomorphic spheres homologous to $S$ (for a suitable $J$ ) is a compact manifold, leading to a contradiction.

We can also reprove an old classic, although as the referee pointed out this does not really constitute a new proof:

Corollary 5 (Eliashberg [6], Gromov [18]) Any weakly symplectically semifillable contact 3-manifold $(M, \xi)$ is tight.

Proof Suppose that $(M, \xi)$ is overtwisted but weakly symplectically semifillable. Let $(X, \omega)$ be a weak symplectic semi-filling of $(M, \xi)$. Cap off all the other components of $\partial X$ as in [10] or [9]. Now apply Lemma 3.1 of either [10] or Stipsicz [22] to attach a cobordism to $(X, \omega)$ to arrive at a homology 3 -sphere contact boundary $\left(M^{\prime}, \xi^{\prime}\right)$ and then use [21] to make sure the new 4-manifold is in fact a strong filling of $\left(M^{\prime}, \xi^{\prime}\right)$. The Legendrian surgeries involved in Lemma 3.1 of [10] can be arranged so as to 
avoid an overtwisted disk in $(M, \xi)$, and the perturbations of the symplectic structure in [21] do not change the contact structure, so we can assume that $\left(M^{\prime}, \xi^{\prime}\right)$ is again overtwisted, but now with a strong symplectic filling.

An overtwisted contact 3-manifold has isotopy classes of tori of torsion greater than 0 (in fact of arbitrarily large torsion). This is because, if we perform arbitrarily many full Lutz twists along a transverse knot in $\left(M^{\prime}, \xi^{\prime}\right)$, we produce a contact structure which is isotopic to $\xi^{\prime}$, using the fact (see Geiges [13]) that full Lutz twists do not change homotopy classes of contact structures and the fact (Eliashberg [5]) that homotopic, overtwisted contact structures are isotopic. Therefore $\left(M^{\prime}, \xi^{\prime}\right)$ contains a copy of $\left(T_{5 / 2}, \eta_{5 / 2}\right)$, which contains a copy of $\left(M_{2}, \xi_{2}\right)$. Corollary 3 then yields our contradiction.

To motivate our last corollary we recall the main results of [11]. There is a natural partial order $\prec$ on the set $\mathcal{C}$ of closed (possibly disconnected, possibly empty) positive contact 3-manifolds: $\left(M_{0}, \xi_{0}\right) \prec\left(M_{1}, \xi_{1}\right)$ if there exists a compact (possibly disconnected) cobordism $X$ from $M_{0}$ to $M_{1}$ equipped with a symplectic form $\omega$ and a Liouville vector field $V$ defined on a neighborhood of $M_{0} \cup M_{1}$, pointing in along $M_{0}$ and out along $M_{1}$, such that $\xi_{i}=\operatorname{ker}\left(\left.l_{V} \omega\right|_{M_{i}}\right)$. (This is what we mean by a "strong symplectic cobordism" and a basic fact is that this relation is reflexive and transitive but not symmetric.) Etnyre and Honda [11] showed the following two facts:

- $(M, \xi) \prec \varnothing$ for every contact 3-manifold $(M, \xi)$.

- For every connected overtwisted contact 3-manifold $\left(M_{o}, \xi_{o}\right)$ and for any other connected contact 3-manifold $(M, \xi)$, we have $\left(M_{o}, \xi_{o}\right) \prec(M, \xi)$.

The connectedness assumption in the second point is related to the absence of models for symplectic 3-handles. Of course 3-handles are involved in any cobordism to $\varnothing$, as in the first point, but those 3-handles are really upside down 1-handles, in the sense that, as elementary cobordisms, if we make the concave end the bottom and the convex end the top, then they are 1-handles.

Now it is reasonable to introduce an equivalence relation on $\mathcal{C}$, whereby $A \sim B$ if $A \prec B$ and $B \prec A$. Then the partial order $\prec$ descends to $\mathcal{C} / \sim$, and one can begin the study of $(\mathcal{C}, \prec)$ with the study of $(\mathcal{C} / \sim, \prec)$. The above results together with the fact that overtwisted contact structures are not fillable mean that there are at least two distinct elements in $\mathcal{C} / \sim$, namely $[\varnothing]$ (precisely the strongly symplectically fillable contact 3 -manifolds), and the equivalence class containing all connected overtwisted contact 3 manifolds, which we will call $\mathcal{O}_{1}$. In addition we have that, for every connected $A \in \mathcal{C}$, $\mathcal{O}_{1} \prec[A] \prec[\varnothing]$. It is also immediate that, if $\mathcal{O}_{i}$ is the equivalence class containing all 
contact 3-manifolds with $i$ components each of which is overtwisted, then $\mathcal{O}_{i} \prec \mathcal{O}_{i-1}$, and that, for any contact 3-manifold $A$ with $i$ components, $\mathcal{O}_{i} \prec[A] \prec[\varnothing]$. Thus we have the following natural questions:

- Is $\mathcal{O}_{i}=\mathcal{O}_{i-1}$ ?

- Are there any tight contact 3 -manifolds in $\mathcal{O}_{1}$ ?

We answer both questions affirmatively:

Corollary 6 For every contact 3-manifold $(M, \xi)$ containing a torus $T$ of torsion greater than 1 , there is a strong symplectic cobordism from $(M, \xi)$ to an overtwisted contact 3-manifold $\left(M^{\prime}, \xi^{\prime}\right)$. If $T$ is separating and $M$ is connected then $M^{\prime}$ will have two components, on each of which $\xi^{\prime}$ is overtwisted.

This answers the first question affirmatively because, as noted in the proof of Corollary 5, overtwisted contact 3-manifolds contain tori of arbitrarily large torsion, which are separating because they are boundaries of solid tori. This answers the second question affirmatively because Giroux [15; 16; 17] and Colin [3] have constructed many examples of weakly fillable, and hence tight, contact 3-manifolds with arbitrarily large torsion. Thus we know the minimal and maximal elements, $\mathcal{O}=\mathcal{O}_{1}$ and $[\varnothing]$, respectively, in $(\mathcal{C} / \sim, \prec)$, and we are left with the obvious question:

Question 7 Are there any elements in $\mathcal{C} / \sim$ other than $\mathcal{O}$ and $[\varnothing]$ ?

As far as we know, this remains open, but the obvious candidates are equivalence classes of tight but not weakly fillable contact 3-manifolds and of contact 3-manifolds containing tori of torsion exactly 1 .

Proof of Corollary 6 By the hypotheses, $(M, \xi)$ contains a copy of $\left(M_{9 / 2}, \xi_{9 / 2}\right)$. We can find real numbers $a, b$ with $0<a<b<9 / 2$ so that $\left(M_{0, a}, \xi_{0, a}\right)$ contains a copy of $\left(M_{1}, \xi_{1}\right)$ in its interior, $\left(M_{a, b}, \xi_{a, b}\right)$ contains a copy of $\left(M_{2}, \xi_{2}\right)$ in its interior, and $\left(M_{b, 9 / 2}, \xi_{b, 9 / 2}\right)$ contains a copy of $\left(M_{1}, \xi_{1}\right)$ in its interior. Attach the cobordism $\left(X_{2}, \omega_{2}\right)$ from Theorem 1 to the $\left(M_{2}, \xi_{2}\right)$ inside $\left(M_{a, b}, \xi_{a, b}\right)$ (extending by the symplectization of $\xi$ on the rest of $(M, \xi))$. The contact 3-manifold $\left(M^{\prime}, \xi^{\prime}\right)$ on the top of the cobordism is then obtained from $(M, \xi)$ by removing the $\left(M_{2}, \xi_{2}\right)$ and replacing with two solid tori. Thus the two remaining copies of $\left(M_{1}, \xi_{1}\right)$ each end up bounding solid tori, so that the solid tori each contain overtwisted meridinal disks. It is clear that if $C$ is separating, then $M^{\prime}$ will be disconnected. 


\section{Proof of Theorem 1}

This proof uses the technique of seeing 4-dimensional symplectic topology through 2-dimensional pictures in moment map images and their generalizations, especially as developed by Symington [23], where a leisurely introduction may be found.

Eliashberg's cobordism in [8] is an $n$-fold cyclic cover of the complement of a neighborhood of a Lagrangian torus in $B^{4}$ equipped with the standard symplectic form. The standard moment map on $\mathbb{R}^{4}$ has image equal to the first quadrant in $\mathbb{R}^{2}$, and this map restricted to $B^{4}$ has image equal to a right-angled triangle with vertices at $(0,0),(1,0)$ and $(0,1)$. Removing a disk from the interior of this triangle corresponds to removing a neighborhood of a Lagrangian torus from $B^{4}$, and then the $n$-fold cyclic cover in question corresponds to the $n$-fold cyclic cover of this punctured right-angled triangle. It is this picture that led the author to the following construction:

Consider the standard moment map $\mu: S^{2} \times S^{2} \rightarrow \mathbb{R}^{2}$ for the standard torus action, translated and rescaled so that $\mu\left(S^{2} \times S^{2}\right)=[-1,1] \times[-1,1]$, and let $\omega$ be the corresponding symplectic form on $S^{2} \times S^{2}$. Recall that the preimage of a point in the interior of the square $[-1,1] \times[-1,1]$ is a Lagrangian torus, that the preimage of a point in the interior of an edge is a circle, that the preimage of a vertex is a point, that the preimage of an entire edge is a symplectic sphere of self-intersection 0 , and that the oriented intersection of two of these spheres (covering two adjacent edges) is +1 . Let $\Gamma$ be the complement in $[-1,1] \times[-1,1]$ of the open disk of radius $1 / 4$ centered at $(0,0)$ and let $X=\mu^{-1}(\Gamma)$. In other words, $X$ is the complement of an open neighborhood of a Lagrangian torus in $S^{2} \times S^{2}$. We will use coordinates $\left(p_{1}, p_{2}\right)$ on $\mathbb{R}^{2}$; recall that these are paired with angular coordinates $\left(q_{1}, q_{2}\right)$ on $S^{2} \times S^{2}$ so that $\omega=d p_{1} \wedge d q_{1}+d p_{2} \wedge d q_{2}$.

Now let $\widetilde{\Gamma}$ be the infinite cyclic cover of $\Gamma$ (with covering map $p: \widetilde{\Gamma} \rightarrow \Gamma$ ) and let $(\tilde{X}, \widetilde{\omega})$ be the corresponding infinite cyclic cover of $(X, \omega)$ (with covering map $\pi: \tilde{X} \rightarrow X)$, so that the natural moment map $\mu \circ \pi$ for $\tilde{X}$ factors through a locally toric fibration $\tilde{\mu}: \tilde{X} \rightarrow \widetilde{\Gamma}$.

Our cobordism $\left(X_{k}, \omega_{k}\right)$ will be a subset of $(\tilde{X}, \tilde{\omega})$ which is the preimage via $\tilde{\mu}$ of a subset $\chi_{k}$ of $\widetilde{\Gamma}$. We describe $\chi_{k}$ in the next few paragraphs. (See Figure 1.)

Consider the following six points in $\Gamma$ :

- $a$ is the intersection of the circle of radius $1 / 4$ centered at $(0,0)$ and the line segment from $(0,0)$ to $(-1 / 2,1 / 4)$.

- $b=(-1 / 2,1 / 4)$

- $c=(-1 / 2,-1)$ 


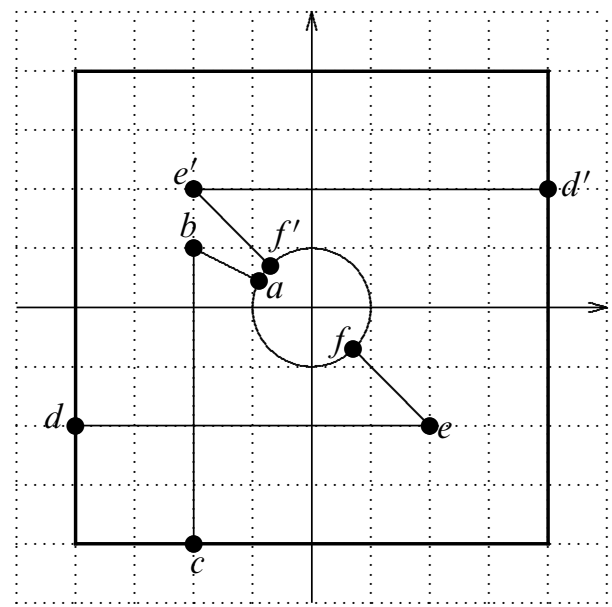

Figure 1: The six points in $\Gamma$ used to construct $\chi_{k}$ (primes on labels indicate the case when $k$ is odd)

- If $k$ is even then $d=(-1,-1 / 2)$, otherwise $d=(1,1 / 2)$.

- If $k$ is even then $e=(1 / 2,-1 / 2)$, otherwise $e=(-1 / 2,1 / 2)$.

- If $k$ is even then $f$ is the intersection of the circle of radius $1 / 4$ centered at $(0,0)$ and the line segment from $(0,0)$ to $(1 / 2,-1 / 2)$, otherwise $f$ is the intersection of the same circle and the line segment from $(0,0)$ to $(-1 / 2,1 / 2)$.

We will want to work with lifts of these points in $\widetilde{\Gamma}$, to which end we establish the following conventions: If we use polar coordinates $(r, \theta)$ on $\Gamma$, where $\theta \in \mathbb{R} / 2 \pi \mathbb{Z}$, then we can naturally lift to coordinates $(r, \tilde{\theta})$ on $\widetilde{\Gamma}$, where $\tilde{\theta} \in \mathbb{R}$. Then, for any point $p \in \Gamma$ and any integer $i, p_{i} \in \widetilde{\Gamma}$ will denote the unique lift of $p$ such that $\widetilde{\theta}\left(p_{i}\right) \in[2 \pi i, 2 \pi(i+1))$. Also, when we speak of straight line segments in $\widetilde{\Gamma}$, we mean arcs that project to straight line segments in $\Gamma$.

Let $n=\lceil k / 2\rceil$. The set $\chi_{k} \subset \widetilde{\Gamma}$ is the compact subset of $\widetilde{\Gamma}$ bounded by:

- the straight line segment $A_{0}$ from $a_{0}$ to $b_{0}$,

- the straight line segment $B_{0}$ from $b_{0}$ to $c_{0}$,

- the part $C$ of the outer (right-angled, polygonal) boundary of $\widetilde{\Gamma}$ going from $c_{0}$ to $d_{n}$,

- the straight line segment $B_{n}$ from $d_{n}$ to $e_{n}$,

- the straight line segment $A_{n}$ from $e_{n}$ to $f_{n}$, and 
- the part $D$ of the inner (round) boundary of $\widetilde{\Gamma}$ going from $f_{n}$ back to $a_{0}$.

This defines the set $\chi_{k}$, and then our cobordism is $X_{k}=\tilde{\mu}^{-1}\left(\chi_{k}\right)$, with $\omega_{k}=\left.\tilde{\omega}\right|_{X_{k}}$. The bottom boundary is $\tilde{\mu}^{-1}(D)$, the sides are $\tilde{\mu}^{-1}\left(A_{0} \cup A_{n}\right)$, and the top boundary is $\tilde{\mu}^{-1}\left(B_{0} \cup B_{n}\right)$. The chain of symplectic spheres is the chain of preimages of the straight line segments of length 2 in $C$. (Note that $C$ starts and ends with segments of length $3 / 2$ and is otherwise composed of $2 k-2$ segments each of length 2 , each meeting the next at a right angle, so that standard toric geometry shows that these spheres satisfy all the advertised properties.) Standard toric geometry also shows that $\tilde{\mu}^{-1}(D) \cong M_{1-p, k+3 / 2+q}$, where $2 \pi p=\tan ^{-1}(1 / 2)$ and $2 \pi q=\tan ^{-1}(1)$, and that $\tilde{\mu}^{-1}\left(B_{0}\right)$ and $\tilde{\mu}^{-1}\left(B_{n}\right)$ are solid tori.

Symington [23] has observed that, if we pick any point $P \in \mathbb{R}^{2}$ and let $W$ be the outward pointing radial vector field centered at $P$, then $P$ lifts to a Liouville vector field $V$ in a toric symplectic 4 -manifold $(X, \omega)$ via the moment map $\mu: X \rightarrow \mathbb{R}^{2}$. If $E$ is an edge of the polygonal moment map image then $V$ will be defined on $\mu^{-1}(E)$ if and only if $V$ is tangent to $E$. (This can be seen by translating the moment map image so that $P=(0,0)$, in which case $V=p_{1} \partial_{p_{1}}+p_{2} \partial_{p_{2}}$. These specific coordinates allow us also to read off the precise contact structure induced by $V$ on any transverse 3-manifold.)

The Liouville vector field advertised in the theorem is then the lift of the radial vector field on $\Gamma$ centered at $(0,0)$, i.e. $V=p_{1} \partial_{p_{1}}+p_{2} \partial_{p_{2}}$. This is transverse to $\tilde{\mu}^{-1}(D)$ and parallel to $\tilde{\mu}^{-1}\left(B_{0}\right)$ and $\tilde{\mu}^{-1}\left(B_{n}\right)$. A direct calculation shows that the induced contact structure on $\tilde{\mu}^{-1}(D) \cong M_{1-p, k+3 / 2+q}$ is precisely $\xi_{1-p, k+3 / 2+q}$. Because the angle $\pi(1-k)$ determines the ray passing through $(-1,2)$ and the angle $\pi(k+3 / 2+q)$ determines the ray passing through $(1,-1)$, a linear transformation in the $S^{1} \times S^{1}$ factor shows that this is contactomorphic to $\left(M_{k+1 / 2}, \xi_{k+1 / 2}\right)$.

Now we construct contact structures $\zeta_{0}$ on $\tilde{\mu}^{-1}\left(B_{0}\right)$ and $\zeta_{n}$ on $\tilde{\mu}^{-1}\left(B_{n}\right)$ which are dominated by $\omega_{k}$ and such that both $\left(\tilde{\mu}^{-1}\left(B_{0}\right), \zeta_{0}\right)$ and $\left(\tilde{\mu}^{-1}\left(B_{n}\right), \zeta_{n}\right)$ are contactomorphic to $\left(T_{1 / 2}, \eta_{1 / 2}\right)$. To this end, we construct vector fields $W_{0}$ and $W_{n}$ along $p\left(B_{0}\right)$ and $p\left(B_{n}\right)$ in $\Gamma$ as follows: Let $W$ be the radial vector field centered at $(0,0)$, let $U_{0}$ be the radial vector field centered at $(3,-1)$ and let $U_{n}$ be the radial vector field centered at $(-1,3)$ if $n$ is even or at $(1,-3)$ if $n$ is odd. Recall that $p\left(B_{0}\right)$ is the line segment from $b$ to $c$. We describe $W_{0}$ as we move from $b$ to $c$. At the beginning, near $b, W_{0}$ is equal to $W$, and then in a short interval $W_{0}$ monotonically interpolates from $W$ to $U_{0}$, and then $W_{0}$ is equal to $U_{0}$ on the rest of $p\left(B_{0}\right)$. This can be done so that, as we move from $b$ to $c, W_{0}$ is turning monotonically counterclockwise relative to the $\left(p_{1}, p_{2}\right)$ coordinate system. Similarly, $p\left(B_{n}\right)$ is the line segment from $e$ to $d$, and $W_{n}$ interpolates from $W$ to $U_{n}$ so that $W_{n}$ monotonically rotates counterclockwise as 
we move from $e$ to $d$. Now lift $W_{0}$ and $W_{n}$ to vector fields $V_{0}$ and $V_{k}$ on $X_{k}$ (as we did for the radial vector fields in the preceding paragraph, except that now these are not Liouville), and let $\zeta_{i}=\left.l_{V_{i}} \omega_{k}\right|_{\tilde{\mu}^{-1}\left(B_{i}\right)}$, for $i=0$ and $n$. Although $V_{i}$ is not Liouville, nevertheless $\zeta_{i}$ will be contact simply because $W_{i}$ is transverse to $p\left(B_{i}\right)$ and rotates monotonically counterclockwise as we move along the length of $p\left(B_{i}\right)$. The fact that $\zeta_{i}$ is dominated by $\omega_{k}$ also follows from the transversality of $W_{i}$ and $p\left(B_{i}\right)$.

As the referee pointed out, the construction above does yield a cobordism in the case $k=1$, which is topologically just a round 2-handle (a 2-handle and a 3-handle). It is not clear what can be proved with such a cobordism, however, because it will not contain a sphere of square 0 .

Thanks The author would like to thank John Etnyre and Andras Stipsicz for helpful comments on an initial version of this paper.

\section{References}

[1] F Bourgeois, V Colin, Homologie de contact des variétés torö̈dales, Geom. Topol. 9 (2005) 299-313 MR2116317

[2] F Bourgeois, Y Eliashberg, H Hofer, K Wysocki, E Zehnder, Compactness results in symplectic field theory, Geom. Topol. 7 (2003) 799-888 MR2026549

[3] V Colin, Une infinité de structures de contact tendues sur les variétés toroïdales, Comment. Math. Helv. 76 (2001) 353-372 MR1839351

[4] F Ding, H Geiges, Symplectic fillability of tight contact structures on torus bundles, Algebr. Geom. Topol. 1 (2001) 153-172 MR1823497

[5] Y Eliashberg, Classification of overtwisted contact structures on 3-manifolds, Invent. Math. 98 (1989) 623-637 MR1022310

[6] Y Eliashberg, Filling by holomorphic discs and its applications, from: "Geometry of low-dimensional manifolds, 2 (Durham, 1989)", LMS Lecture Notes 151, Cambridge Univ. Press, Cambridge (1990) 45-67 MR1171908

[7] Y Eliashberg, Topological characterization of Stein manifolds of dimension $>2$, Internat. J. Math. 1 (1990) 29-46 MR1044658

[8] Y Eliashberg, Unique holomorphically fillable contact structure on the 3-torus, Internat. Math. Res. Notices (1996) 77-82 MR1383953

[9] Y Eliashberg, A few remarks about symplectic filling, Geom. Topol. 8 (2004) 277-293 MR2023279

[10] J B Etnyre, On symplectic fillings, Algebr. Geom. Topol. 4 (2004) 73-80 MR2023278 
[11] J B Etnyre, K Honda, On symplectic cobordisms, Math. Ann. 323 (2002) 31-39 MR1906906

[12] D T Gay, Explicit concave fillings of contact three-manifolds, Math. Proc. Cambridge Philos. Soc. 133 (2002) 431-441 MR1919715

[13] H Geiges, Contact geometry, from: "Handbook of differential geometry. Vol. II", Elsevier/North-Holland, Amsterdam (2006) 315-382 MR2194671

[14] P Ghiggini, Ozsváth-Szabó invariants and fillability of contact structures, Math. Z. 253 (2006) 159-175 MR2206641

[15] E Giroux, Une structure de contact, même tendue, est plus ou moins tordue, Ann. Sci. École Norm. Sup. (4) 27 (1994) 697-705 MR1307678

[16] E Giroux, Une infinité de structures de contact tendues sur une infinité de variétés, Invent. Math. 135 (1999) 789-802 MR1669264

[17] E Giroux, Structures de contact en dimension trois et bifurcations des feuilletages de surfaces, Invent. Math. 141 (2000) 615-689 MR1779622

[18] M Gromov, Pseudoholomorphic curves in symplectic manifolds, Invent. Math. 82 (1985) 307-347 MR809718

[19] P Lisca, A I Stipsicz, Symplectic fillability and Giroux torsion arXiv: math.SG/0604268

[20] D McDuff, The structure of rational and ruled symplectic 4-manifolds, J. Amer. Math. Soc. 3 (1990) 679-712 MR1049697

[21] H Ohta, K Ono, Simple singularities and topology of symplectically filling 4-manifold, Comment. Math. Helv. 74 (1999) 575-590 MR1730658

[22] A I Stipsicz, On the geography of Stein fillings of certain 3-manifolds, Michigan Math. J. 51 (2003) 327-337 MR1992949

[23] M Symington, Four dimensions from two in symplectic topology, from: "Topology and geometry of manifolds (Athens, GA, 2001)", Proc. Sympos. Pure Math. 71, Amer. Math. Soc., Providence, RI (2003) 153-208 MR2024634

[24] C H Taubes, The Seiberg-Witten invariants and symplectic forms, Math. Res. Lett. 1 (1994) 809-822 MR1306023

[25] A Weinstein, Contact surgery and symplectic handlebodies, Hokkaido Math. J. 20 (1991) 241-251 MR1114405

Department of Mathematics and Applied Mathematics, University of Cape Town

Private Bag X3, Rondebosch 7701, South Africa

dgay@maths.uct.ac.za

Proposed: Yasha Eliashberg

Seconded: Peter Ozsváth, Tomasz Mrowka

Received: 22 June 2006

Accepted: 13 October 2006

Geometry $8 \mathcal{T}$ Topology, Volume 10 (2006) 